\title{
Mucosal Melanoma of the Head and Neck by AJCC v7 Stage
}

National Cancer Institute

\section{Source}

National Cancer Institute. Mucosal Melanoma of the Head and Neck by A/CC v7 Stage. NCI Thesaurus. Code C133186.

A term that refers to the staging of mucosal melanoma of the head and neck according to the American Joint Committee on Cancer, 7th edition. No prognostic stage grouping is proposed in the 8th edition. 